\title{
Photon detection by current-carrying superconducting film: A time-dependent Ginzburg-Landau approach
}

\author{
A. N. Zotova and D. Y. Vodolazov * \\ Institute for Physics of Microstructures, Russian Academy of Sciences, 603950, Nizhny Novgorod, GSP-105, Russia
}

(Dated: July 3, 2018)

\begin{abstract}
We study dynamics of the order parameter in a superconducting film with transport current after absorption of a single photon. The system from the time-dependent Ginszburg-Landau equation, Poisson's equation for an electrical potential and the heat diffusion equation was solved numerically. For each photon energy in the absence of fluctuations, there exists a corresponding threshold current below which the superconducting state is stable and no voltage appears between the ends of the film. At larger currents the superconducting state collapses starting from the appearance of a vortex-antivortex pair in the center of the region with suppressed superconducting order parameter which has been created by the absorbed photon. Lorentz force causes motion of these vortices that heats the film locally and gives rise to a normal domain. When biased with the fixed current, the film latches in the normal state. In the regime when current via superconductor may change, which is more relevant for experiments, the normal domain exists only for a short time resulting in the voltage pulse with the duration controlled by the kinetic inductance of the superconducting film.
\end{abstract}

PACS numbers: 74.25.Op, 74.20.De, 73.23.-b

\section{INTRODUCTION}

Despite the large number of both experimental (see for example [1-7] and references therein) and theoretical [811] works on the superconductive single-photon detectors (SSPD) still there are some questions about mechanism of photon detection by superconductive film carrying the transport current. The original understanding of the detection mechanism is following - after absorption of the single photon the hot spot is formed in the superconducting film. It locally destroys superconductivity and leads to concentration of the current density outside the hot spot due to decrease of the effective width of the film [8]. If transport current is close to the depairing current then the current density outside the hot spot area may exceed the depairing current density and the superconducting state becomes unstable, leading to the voltage response.

The quantitative analysis of initial stage of the hot spot formation in the existing theoretical models is based on the solution of the diffusion equation for nonequilibrium quasiparticles [8 [10]. It was postulated that when the number of the nonequilibrium quasiparticles in the hot spot exceeds some critical value this region could be considered as a normal one and the superconducting current is forced to flow around it. In the refined model [9] it was supposed that even partial suppression of the superconducting order parameter in the hot spot leads to current enhancement outside that region and to instability of the superconducting state and formation of the normal domain. The further evolution of the normal domain is usually studied by using the heat diffusion equation for effective temperature of quasiparticles coupled with equation describing the embedding circuit [11 13].

*Electronic address: vodolazov@ipm.sci-nnov.ru
In the majority of previous models it was implicitly assumed that the magnitude of the superconducting order parameter $|\Delta|$ changes instantly in time if local temperature $T(\vec{r}, t)>T_{c}$ or local superconducting current density $j(\vec{r}, t)$ exceeds depairing current density $j_{\text {dep }}$. But it is well know that $|\Delta|$ has finite relaxation time $\tau_{|\Delta|}$ and in some cases $\tau_{|\Delta|}$ could be comparable with electronphonon inelastic relaxation time $\tau_{e-p h}$ [14]. Because energy relaxation of nonequilibrium quasiparticles in the hot spot occurs on the same time scale (or even much shorter due to diffusion of the quasiparticles) it is clear that finite $\tau_{|\Delta|} \neq 0$ should influence the photon detection process [15.

Another interesting and unresolved question is what kind of instability of the superconducting state occurs due to appearance of the hot spot region. Is it gradual suppression of the order parameter outside the hot spot due to current concentration [8, 15] or nucleation of the vortex-antivortex pair inside the hot spot [16]?

In our work we use the simplest approach where effect of finite $\tau_{|\Delta|}$ is taken into account and stability of the superconducting state is analyzed self-consistently. The dynamics of the superconducting order parameter is studied on the basis of the time-dependent GinzburgLandau equation. This equation is coupled with the heat diffusion equation for the effective temperature of the quasiparticles and Poisson's equation for the electrical potential. We consider the current bias regime as well as the regime when current via superconductor may change due to presence of the shunt resistance and take into account the finite kinetic inductance of the film.

Within this model we show that incoming photon creates the finite size region with partially suppressed order parameter. We find that even for infinite superconducting film such a state becomes unstable without any fluctuations with respect to appearance of the vortexantivortex pair (or single vortex if photon is absorbed on 
the edge of the film) at threshold current less than depairing current. Motion of the vortex and antivortex in opposite directions under the Lorentz force heats the sample (if the threshold current is not too small). As a result, the normal domain appears which either expands over the whole film (current bias regime) or shrinks and disappears (when current via superconductor may change) resulting in the voltage pulse. Our result supports the hypothesis of Ref. [16] that the single photon can nucleate the vortex-antivortex pair in current-carrying superconductor and their motion provides the voltage pulse which could be detected. Our model also confirms the experimentally observed smeared red boundary in the singlephoton detection.

The paper is organized as follows. In section II we present the theoretical model. The results of the numerical calculations and simple analytical estimations are given in Section III. In section IV we discuss the relation of our results with an experiment and in section $\mathrm{V}$ we present our conclusions.

\section{MODEL}

In our work we do not study the initial part of the detection process when the single photon is absorbed by the electron. We use approach of the effective temperature [17], which is valid when the thermalization time (which is proportional to the electron-electron inelastic relaxation time $\tau_{e-e}$ ) is shorter than the inelastic relaxation time due to electron-phonon interactions $\tau_{e-p h}$. We assume that during initial time interval $\sim \tau_{e-e}$ after absorption of the photon the electron-electron interactions creates hot spot with radius $R_{\text {init }} \sim L_{e-e}=\left(D \tau_{e-e}\right)^{1 / 2}$ ( $\mathrm{D}$ is a diffusion constant) and with local temperature $T_{0}+\Delta T\left(T_{0}\right.$ is a bath temperature) where $\Delta T$ is determined from the energy conservation

$$
2 \pi \hbar c / \lambda=\Delta T \pi R_{\text {init }}^{2} d C_{v}
$$

Here $\lambda$ is a wavelength of the electromagnetic radiation, $\hbar$ is a Planck constant, c is a speed of light, $\mathrm{d}$ is a thickness of the film and $C_{v}$ is a heat capacity of the quasiparticles (for simplicity we take $C_{v}$ as in the normal state at $T=T_{c}$ ).

Time and space evolution of the temperature in the superconducting film we find from the heat diffusion equation

$$
\frac{\partial T}{\partial t}=D\left(\frac{\partial^{2} T}{\partial x^{2}}+\frac{\partial^{2} T}{\partial y^{2}}\right)+\frac{\rho_{n} j_{n}^{2}}{C_{v}}-\frac{T-T_{0}}{\tau_{e-p h}}
$$

where $\rho_{n}$ is a normal state resistivity, $j_{n}=-\nabla \varphi / \rho_{n}$ is a normal current density and $\varphi$ is a electrostatic potential. Here we assume that the phonons are in equilibrium with the bath and energy relaxation occurs due to interaction with phonons. Our calculations show, that initial destruction of superconductivity occurs on timescale shorter than $\tau_{e-p h}$ and therefore at initial stage of dynamical response of $|\Delta|$ one may neglect the heating of phonons (in our model we neglect possibility of the phonon heating during initial $t \lesssim \tau_{e-e}$ stage of hot spot formation).

To study the dynamics of the order parameter $\Delta=$ $|\Delta| e^{i \phi}$ we use the time-dependent Ginzburg-Landau equation

$$
\begin{aligned}
\frac{\pi \hbar}{8 k_{B} T_{c}}\left(\frac{\partial}{\partial t}-\frac{i 2 e \varphi}{\hbar}\right) \Delta & =\xi_{G L}(0)^{2}\left(\frac{\partial^{2} \Delta}{\partial x^{2}}+\frac{\partial^{2} \Delta}{\partial y^{2}}\right) \\
& +\left(1-\frac{T}{T_{c}}-\frac{|\Delta|^{2}}{\Delta_{G L}(0)^{2}}\right) \Delta
\end{aligned}
$$

where $\xi_{G L}(0)=\left(\pi \hbar D / 8 k_{B} T_{c}\right)^{1 / 2}$ and $\Delta_{G L}(0)=$ $4 k_{B} T_{c} u^{1 / 2} / \pi(u \simeq 5.79$ - see [18] $)$ are the zero temperature Ginzburg-Landau coherence length and the order parameter respectively. Characteristic time relaxation of the order parameter described by Eq. (3) is $\tau_{|\Delta|}=\pi \hbar / 8 k_{B}\left(T_{c}-T\right)$. Although Eq. (3) is quantitatively valid only near critical temperature of the superconductor (at $T \gtrsim 0.9 T_{c}$ when $\tau_{e-e} \ll \tau_{e-p h}$ and $\left.\tau_{e-e} \ll \tau_{|\Delta|}\right)$ we use it to model the dynamics of the superconducting condensate at lower temperatures to find some qualitative results.

We should complete Eqs. (2-3) by equation for the electric potential $\varphi$ which comes from the conservation of the full current $\operatorname{div}\left(j_{s}+j_{n}\right)=0$

$$
\Delta \varphi=\rho_{n} \operatorname{div}\left(j_{s}\right)
$$

here $j_{s}=\operatorname{Imag}\left(\Delta^{*} \nabla \Delta\right) /\left(4 e k_{B} T_{c} \rho_{n}\right)$.

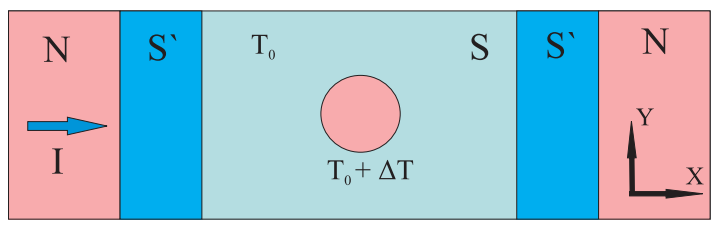

(a)

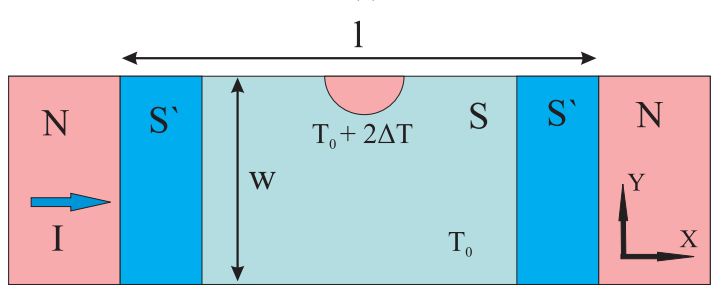

(b)

FIG. 1: The model geometry: the superconductive film is placed between two normal bulk contacts; (a) - the photon is absorbed in the center of the film, (b) - the photon is absorbed on the edge of the film.

To model the response of the superconducting film after absorption of the single photon we consider the model geometry which is present in Fig. 1. We need the normal contacts $\left(\Delta=0, \partial \varphi / \partial x=-\rho_{n} I /(w d)\right.$ at $\left.x= \pm l / 2\right)$ to inject the current to the superconducting film in our numerical calculations and which are kept at the bath temperature $T_{0}\left(\left.T\right|_{x= \pm l / 2}=T_{0}\right)$. The current and heat do 
not flow through the lateral edges of the film $(\partial T / \partial y=0$, $\partial \varphi / \partial y=0, \partial \Delta / \partial y=0$ at $y= \pm w / 2)$. To neglect the influence of the N-S boundaries (for example the motion of the NS boundary) on the dynamical processes in the superconducting film we artificially enhance the superconducting order parameter in the regions marked by dark blue color in Fig. 1 by introducing locally higher $T_{c}$ (the width of these regions is larger than the penetration depth of the electric field from the normal contact and is equal to $\left.5 \xi_{G L}(0)\right)$.

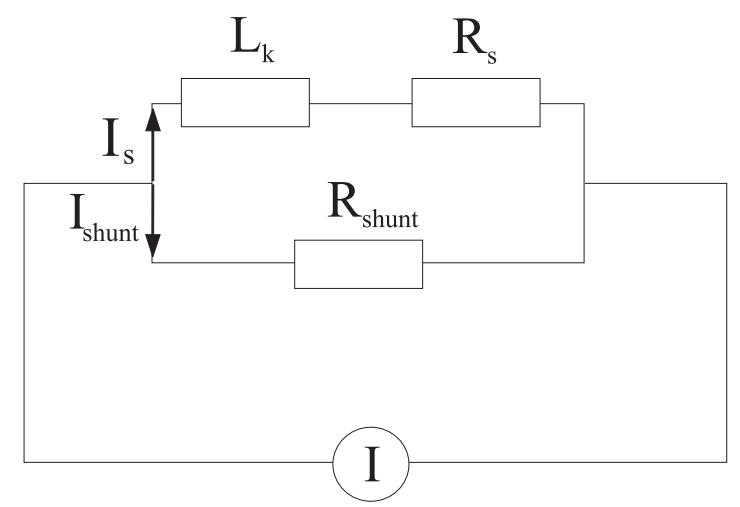

FIG. 2: The equivalent scheme of the superconducting detector. The superconductor is modelled by kinetic inductance $L_{k}$ and resistance $R_{s}$ which appeared due to absorbing the photon. The shunt has resistance $R_{\text {shunt }}$.

To model real experiments we consider electrical scheme which is shown in Fig. 2. Here $L_{k}$ is the kinetic inductance of the superconducting film, $R_{s}$ corresponds to the resistance of the superconductor in the resistive state (in the model geometry - see Fig. 1 ) and $R_{\text {shunt }}$ is the shunting resistance. For this case we have to find current $I_{s}$ which flows via superconductor from the solution of the following equation

$$
\frac{L_{k}}{c^{2}} \frac{d I_{s}}{d t}=\left(I-I_{s}\right) R_{\text {shunt }}-V_{s}
$$

where the voltage drop over superconductor $V_{s}$ (over the blue region in Fig. 1) should be found from the solution of Eq. (4) with boundary condition $\partial \varphi /\left.\partial x\right|_{x= \pm L / 2}=$ $-\rho_{n} I_{s} /(w d)$.

In numerical calculations we use the dimensionless units. The order parameter is scaled in units of $\Delta_{G L}(0)$, temperature is in units of $T_{c}$ and coordinate is in units of $\xi_{G L}(0)$. Time is scaled in units of $\tau_{0}=\pi \hbar / 8 k_{B} T_{c} u$, electrostatic potential is in units of $\varphi_{0}=\hbar / 2 e \tau_{0}$ and current density is in units of $j_{0}=\hbar / 2 e \rho_{n} \tau_{0} \xi_{G L}(0)$ (depairing current density in these units is $j_{\text {dep }} / j_{0}=(4 / 27)^{1 / 2}(1-$ $\left.T / T_{c}\right)^{3 / 2}$.

To solve Eqs. $(2,3,5)$ numerically we use Euler method and to solve Eq. (4) - Fourier analysis and cyclic reduction method. In numerical calculations we first apply the finite current and wait until all relaxation processes connected with the current induced suppression of the order

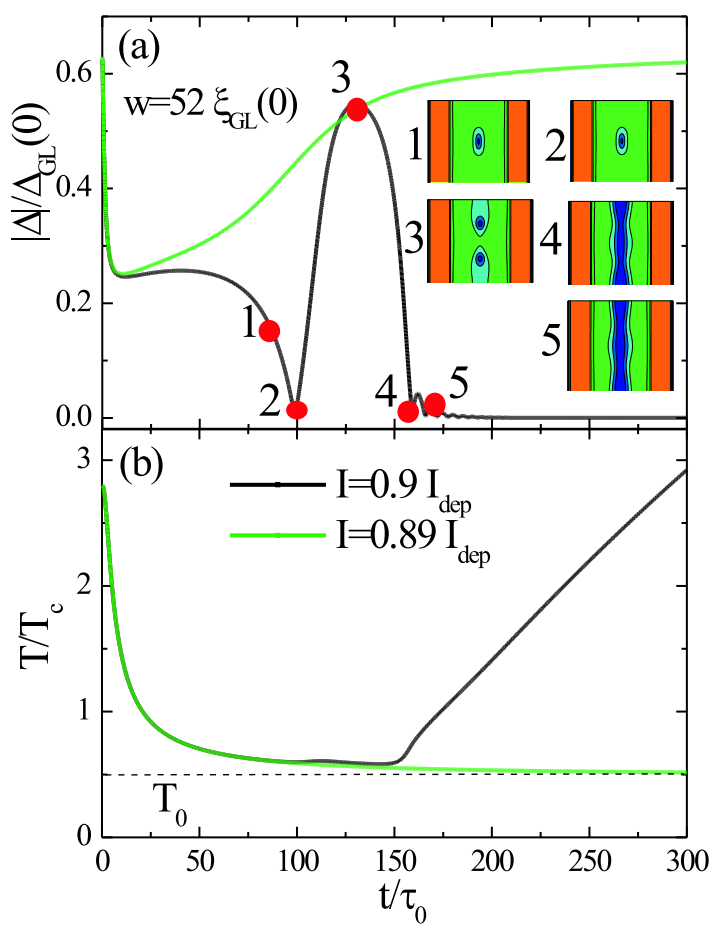

FIG. 3: Time dependence of the magnitude of the order parameter (a) and temperature (b) in the center of the hot spot (which coincide with a center of the film) for two values of the transport current $I=0.89 I_{d e p}$ and $I=0.9 I_{d e p}$. The width of the film $w=52 \xi_{G L}(0)$, the local initial increase of the temperature $\Delta T=2.3 T_{c}(\lambda \simeq 6.5 \mu \mathrm{m})$. In the inset we show contour plots of the magnitude of the order parameter in the film at different times marked by the numbers on the black solid curve.

parameter stops. Than at some moment of time we instantly increase the temperature by $\Delta T$ in the circle or semicircle area inside the superconductor (see Fig. 1) and studied the dynamical response of the system. The parameters of the film are: length $l=60 \xi_{G L}(0)$ and width $\mathrm{w}$ is varied from $13 \xi_{G L}(0)$ up to $78 \xi_{G L}(0)$.

In our calculations we use parameters typical for $\mathrm{NbN}$ SSPD [8]: $C_{v}=2.4 \mathrm{~mJ} \mathrm{~cm}^{-3} \mathrm{~K}^{-1}, \tau_{e-e}=7 \mathrm{ps}, D=$ $0.45 \mathrm{~cm}^{2} / \mathrm{s}, \xi_{G L}(0)=7.5 \mathrm{~nm}, T_{c}=10 K, \tau_{e-p h}=17 \mathrm{ps}$. At these parameters $L_{e-e} \simeq 18 \mathrm{~nm}$ and $\tau_{0}=0.052 \mathrm{ps}$. In test calculations we consider two values for $R_{\text {init }}=18 \mathrm{~nm}$ and $R_{\text {init }}=9 \mathrm{~nm}$ which are close to $L_{e-e}$ and found that the results (in particular the value of the threshold when the voltage appears) differ only slightly. The presented below results are obtained with $R_{\text {init }}=9 \mathrm{~nm}$. For this radius and thickness of the film $d=4 \mathrm{~nm}$ the range of $\Delta T=0.3-12.8 T_{c}$ corresponds to the wavelengths $\lambda \simeq$ $1.3-50 \mu \mathrm{m}$. The bath temperature $T_{0}$ is equal to $T_{c} / 2$. 


\section{RESULTS}

\section{A. Regime with constant current}

At first we consider current bias regime (when $I_{s}=I$ and $I_{\text {shunt }}=0$ in Fig. 2 for $R_{\text {shunt }} \rightarrow \infty$ ). In Fig. 3 we present time dependence of the magnitude of the order parameter and effective temperature of quasiparticles in the center of the film with width $w=52 \xi_{G L}(0)$ and for the situation depicted in Fig. 1(a) for two close values of the transport current. Notice that suppression of the order parameter in the center of the hot spot needs finite time (see Fig. 3(a)). During this time the local temperature in the center of hot spot decreases (see Fig. $3(b)$ ) due to diffusion of the nonequilibrium quasiparticles and energy transfer to phonons. When the current is smaller than the threshold value (we call it the detecting current $I_{d}$ ) the order parameter after reaching some minimal value starts to grow. In this case the time averaged voltage response is zero. The larger current destroys superconducting state. In this case $|\Delta|$ oscillates in the center of the hot spot with the amplitude which decays in time. Each oscillation of $|\Delta|$ corresponds to nucleation of one vortex-antivortex pair. Motion of the vortex/antivortex in opposite directions (see inset in Fig. $3(\mathrm{a})$ ) heats the superconductor via Joule dissipation and the local temperature increases. It results in appearance of the growing resistive domain (see inset in Fig. 3(a)) in the regime of the constant current at chosen parameters.

In Figs. 4-5 we show the time evolution of the order parameter in the films with smaller width at $I>I_{d}$ and one can see qualitatively the same scenario of the order parameter dynamics. The dependence of the detecting current on $\Delta T$ (i.e. on the energy of the absorbed photon) and width of the film is shown in Fig. 6. The detecting current decreases with the increase of the photon energy and its value depends on the position where the photon is absorbed (in the center or on the edge of the film). For fixed photon energy the ratio $I_{d} / I_{d e p}$ initially grows with increasing width of the film and than saturates for large w (see Fig. 6(b)).

We shall note that for high energy photons (large $\Delta T$ ) and relatively narrow film the detecting current is much smaller than depairing current (see Fig. 6(a)). In this case the Joule dissipation could be weak, the normal domain does not appear and superconductivity recovers after nucleation of several vortex-antivortex pairs in the hot spot area. For film with $w=13 \xi_{G L}(0)$ and $\Delta T=12.8 T_{c}$ the normal domain appears only at $I>0.36 I_{\text {dep }}\left(I_{d} \simeq 0.23 I_{\text {dep }}\right.$ - see Fig. 6(a)) which is close to the value of the current when the heat dissipation and heat removal are equal to each other

$$
\frac{\rho_{n} j_{\text {heat }}^{2}}{C_{v}}=\frac{T_{c}-T_{0}}{\tau_{e-p h}}
$$

and for our choice of parameters $I_{\text {heat }}=j_{\text {heat }} w d \simeq$ $0.23 I_{\text {dep }}$.

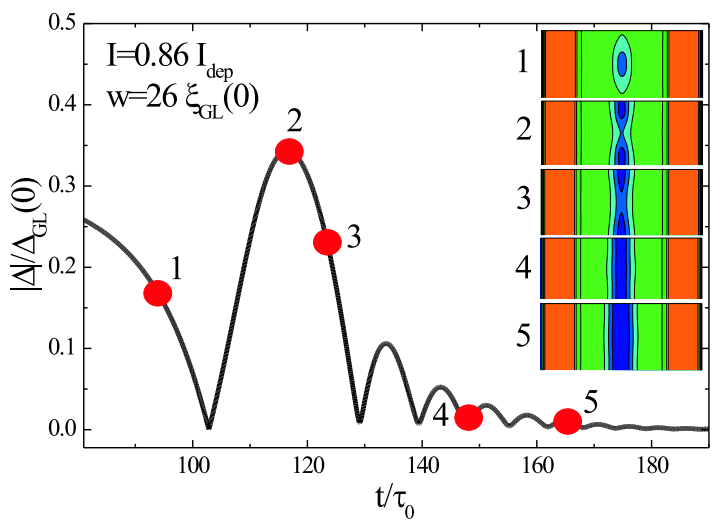

FIG. 4: Time dependence of the magnitude of the order parameter in the center of the hot spot (which coincides with the center of the film). The width of the film $w=26 \xi_{G L}(0)$, the bias current $\mathrm{I}=0.86 I_{d e p}$, the local initial increase of the temperature $\Delta T=2.3 T_{c}(\lambda \simeq 6.5 \mu \mathrm{m})$. The inset show contour plots of the magnitude of the order parameter in the film at different times marked by the numbers on the black solid curve.

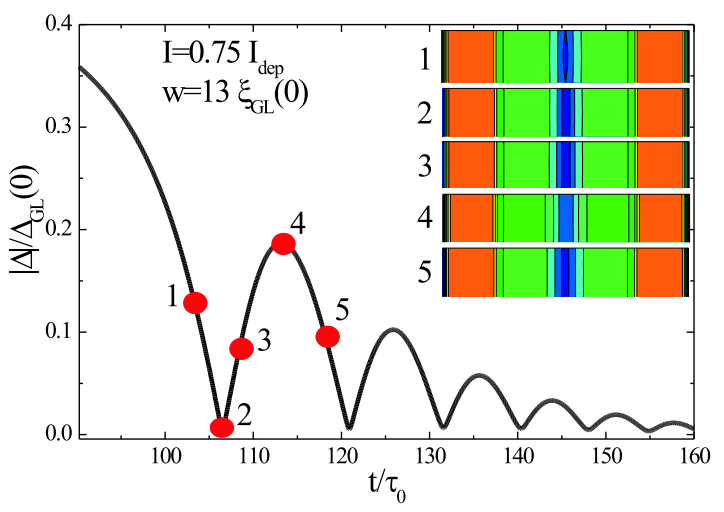

FIG. 5: Time dependence of the magnitude of the order parameter in the center of the hot spot (which coincides with the center of the film). The width of the film $w=13 \xi_{G L}(0)$, the bias current $I=0.75 I_{d e p}$, the local initial increase of the temperature $\Delta T=2.3 T_{c}(\lambda \simeq 6.5 \mu \mathrm{m})$. The inset show contour plots of the magnitude of the order parameter in the film at different times marked by the numbers on the black solid curve.

According to our numerical calculations the voltage response appears when the vortex-antivortex pair is nucleated in the center of the hot spot. To get insight why it occurs we consider the following simple model. Let us model the region with suppressed $|\Delta|$ in the hot spot by circle of radius $R$ and we assume that $|\Delta|$ is spatially uniform and has value $\Delta_{i n}$ inside the circle and in the rest of the infinite thin superconducting film $|\Delta|=\Delta_{\text {out }}>\Delta_{\text {in }}$. We are interested how transport current is distributed in such a superconducting system and when superconducting vortex free state becomes unstable. For simplicity we neglect the proximity effect (which is reasonable when $R \gg \xi$ ) and use the London model 

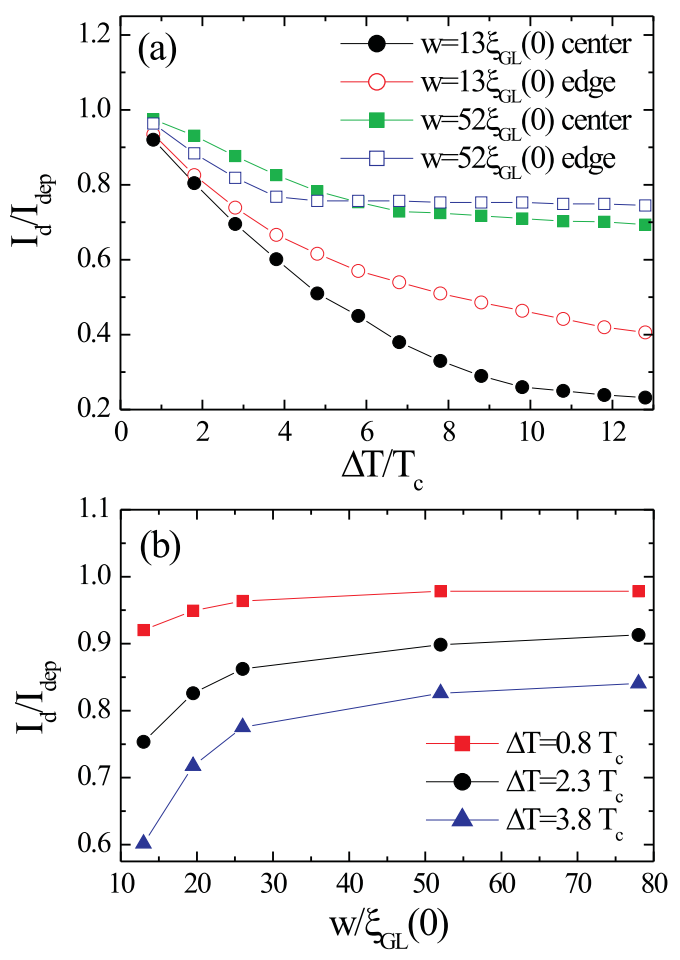

FIG. 6: (a) The dependence of the detecting current on the instant increase of the temperature in the circle with radius $R_{\text {init }}$ for narrow and wide films with two positions of the photon absorbtion (in the center and on the edge of the film). (b) The dependence of the detecting current on the width of the superconductive film for three values of $\Delta T / T_{c}=0.8,2.3$, 3.8 , corresponding to three wavelengths of the electromagnetic radiation $\lambda=18.8,6.5$ and $3.9 \mu \mathrm{m}$ respectively (photon is absorbed in the center of the film).

$j_{s}=|\Delta|^{2} \nabla \phi /\left(4 e k_{B} T_{c} \rho_{n}\right)$. Distribution of the superconducting current can be found from the current conservation $\operatorname{divj}_{s}=0$. As a result we obtain that inside the circle the supervelocity $v \sim \nabla \phi$ is larger than $v$ in infinity

$$
v_{i n}=\frac{2 v_{\infty}}{1+\gamma^{2}}
$$

$\left(\gamma=\Delta_{\text {in }} / \Delta_{\text {out }}\right)$ and it is locally enhanced outside the circle

$$
v_{\text {out }}(r)=v_{\infty}\left(1+\frac{R^{2}}{r^{2}} \frac{1-\gamma^{2}}{1+\gamma^{2}}\right), r>R
$$

where the distance is measured from the center of the circle and we present result at angle $\alpha=\pi / 2$ between the direction of the current and radial vector in polar system of coordinate.

One may find corrections to Eqs. $(7,8)$ for film with finite width in the limit when $2 R / w \ll 1$ and the circle is placed in the center of the film (see Fig. 1(a)). Assume that for finite film with $2 R / W \ll 1$ the Eqs. $(7,8)$ are approximately valid, but coefficient $v_{\infty}$ we replace by unknown $v^{*}$ which we find from the conservation of the full current

$$
\begin{aligned}
& I \sim \Delta_{\text {out }}^{2} v_{\infty} w d=2 d \int_{0}^{R} \frac{2 \Delta_{\text {in }}^{2} v^{*}}{1+\gamma^{2}} d y+ \\
& +2 d \int_{R}^{w / 2} \Delta_{\text {out }}^{2} v^{*}\left(1+\frac{R^{2}}{y^{2}} \frac{1-\gamma^{2}}{1+\gamma^{2}}\right) d y
\end{aligned}
$$

As a result we find

$$
v^{*}=v_{\infty} /\left(1-\left(\frac{2 R}{w}\right)^{2} \frac{1-\gamma^{2}}{1+\gamma^{2}}\right)
$$

Note that Eq. (10) is also valid (with replacement $2 R / w \rightarrow R / w)$ for the case when semicircle of radius $\mathrm{R}$ with suppressed $|\Delta|=\Delta_{i n}$ is placed on the edge of the film (see Fig. 1(b)). We have to stress that coefficient in front of term $(2 R / w)^{2}$ in Eq. (10) is approximately valid (up to coefficient of order of unity) and correct value should be found from the expansion of the exact result in series with small parameter $2 R / w$.

Because $\Delta_{\text {in }}<\Delta_{\text {out }}$ and $v_{\text {in }}>v_{\text {out }}$ the superconducting Meissner (vortex free) state first becomes unstable inside the circle(semicircle). Using value of the critical supervelocity $v_{c} \sim|\Delta|$ for instability of spatially uniform superconducting state which follows from stationary Eq. (3) we find (by equalizing $v_{i n}=v_{c}$ and using Eq. (10))

$$
\frac{I_{\text {pair }}}{I_{\text {dep }}}=\frac{\gamma\left(1+\gamma^{2}\right)}{2}\left(1-\left(\frac{2 R}{w}\right)^{2} \frac{1-\gamma^{2}}{1+\gamma^{2}}\right)
$$

The current $I_{\text {pair }}$ is our estimation for the current when the vortex-antivortex pair is nucleated inside the circle (with replacement $2 R / w \rightarrow R / w$ it corresponds to the threshold current when single vortex is nucleated in the center of the semicircle on the edge of the film). It is worth to mention here that it is not the current when the resistive state appears in the sample, because to escape the circle(semicircle) the vortex and antivortex should overcome the energy barrier connected with jump in $|\Delta|$. To estimate this critical current we assume that the vortices may leave the circle(semicircle) when the supervelocity (averaged over finite region $\sim \xi(T)$ near the edge of the circle) is equal to $v_{c}$. Using Eqs. $(8,10)$ it is easy to find that

$\frac{I_{r e s}}{I_{d e p}}=\left(1-\left(\frac{2 R}{w}\right)^{2} \frac{1-\gamma^{2}}{1+\gamma^{2}}\right) /\left(1+\frac{R}{R+\xi(T)} \frac{1-\gamma^{2}}{1+\gamma^{2}}\right)$

One can see that $I_{\text {pair }} \leq I_{\text {res }}$ (they are equal when $\Delta_{\text {in }}=\Delta_{\text {out }}$ and $\gamma=1$ ). Both critical currents decrease with decreasing $\Delta_{i n}$ and $I_{\text {pair }}=0$ and $I_{r e s}=I_{d e p}(1-$ $\left.4 R^{2} / w^{2}\right) / 2$ when $R \gg \xi(T)$ and $\gamma=0$ (it corresponds to the normal state of the circle).

To complete the analytical analysis we have to correlate the radius of the region with suppressed $|\Delta|$ and $\Delta_{i n}$ with energy of the incoming photon. Let us assume that 
the spatial and time dependence of the temperature after photon absorbtion is described by the following expression

$$
T(r, t)=\frac{\beta}{4 \pi D t} e^{-r^{2} / 4 D t}+T_{0}
$$

which is solution of the Eq. (2) with replacement of heating term by $\beta \delta(t) \delta(\vec{r})\left(\beta=2 \pi \hbar c / \lambda C_{v} d\right)$ which describes the energy delivered by photon to the quasiparticles at the moment $\mathrm{t}=0$ and in the point $\mathrm{r}=0$ (we also neglect the last term in Eq. (2) because we are interested in time interval of about $\tau_{|\Delta|} \ll \tau_{e-p h}$ after photon absorption).

Local enhancement of temperature leads to suppression of the order parameter in the hot spot. We may estimate it by using Eq. (3) where we neglect for simplicity the term with the second derivative

$$
\tau_{|\Delta|}(0) \frac{\partial|\Delta|}{\partial t}=\left(1-\frac{T}{T_{c}}-\frac{|\Delta|^{2}}{\Delta_{G L}(0)^{2}}\right)|\Delta|
$$

One can see that while left hand side of Eq. (14) is negative the order parameter decreases. Because $T$ is maximal in the center of hot spot and decreases in time it is reasonable to suppose that the order parameter stops to decrease when $T=T_{c}$ in the center of the hot spot. Using Eq. (13) we find that it occurs at

$$
\delta t=\frac{\beta}{4 \pi D\left(T_{c}-T_{0}\right)} \simeq \frac{\Delta T}{T_{c}} \frac{\tau_{|\Delta|}\left(T_{0}\right)}{4}
$$

where we used Eq. (1) to express $\beta=\Delta T \pi R_{\text {init }}^{2}$ and $R_{\text {init }}=1.2 \xi_{G L}(0)$ via parameters of our numerical model. Using this result and Eq. (13) we may estimate the size of the region where the order parameter is suppressed

$$
R \simeq 2 \sqrt{D \delta t}=\sqrt{\frac{\beta}{\pi\left(T_{c}-T_{0}\right)}} \simeq \xi(T) \sqrt{\frac{\Delta T}{T_{c}}}
$$

From Eq. (13) one may find suppression of $|\Delta|$ in hot spot at $r<R$ by moment $t=\delta t$. Below we assume that $\Delta_{i n}=|\Delta|(r=\xi, t=\delta t)$. Using Eqs. $(13,14)$ we find

$$
\begin{aligned}
& \gamma=\frac{\Delta_{\text {in }}}{\Delta_{\text {out }}} \simeq \exp \left(-\frac{1}{\tau_{|\Delta|}(0)} \int_{0}^{\delta t} \frac{T(\xi, t)}{T_{c}} d t\right) \\
& \simeq \exp \left(-\frac{\beta}{4 \pi \xi_{G L}(0)^{2} T_{c}} \ln \left(\frac{\beta}{\pi \xi_{G L}(0)^{2} T_{c}}\right)\right)
\end{aligned}
$$

which is approximately valid for photons with $\left(\beta / \pi \xi_{G L}(0)^{2} T_{c} \simeq \Delta T / T_{c} \gtrsim 1\right)$.

For photon absorbed on the edge (which creates the semicircle) above results are also valid with the replacement of $\beta$ by $2 \beta$. Finite width of the film does not affect above results too much while $w \gg 2 R$ (or $w \gg R$ for the semicircle) due to exponential decay of temperature at $r>R$.

Combination of Eqs. $(16,17)$ and Eqs. $(11,12)$ qualitatively explains our numerical results. First of all with increase of the photon energy $R$ increases and $\gamma$ decreases providing decrease of $I_{r e s}$ and $I_{\text {pair }}$ (compare with Fig. $6(\mathrm{a}))$.

Secondly, above analytical results also explain decrease of detecting current with decreasing width of the sample (compare Fig. 6 and Eq. (12)). Thirdly, when the photon is absorbed on the edge of the film it creates the semicircle with larger radius $R^{\prime}=\sqrt{2} R$ (in comparison with photon absorbed in the center). It results in larger detecting current than $I_{d}$ for photon absorbed in the center of the film (see Eqs. $(11,12$ ) with replacement $2 R / w$ by $R^{\prime} / w$ and for high energy photons when $\gamma \ll 1$ ). Note that the effect is stronger for films with smaller width (compare with Fig. 6(a)).

For photons of relatively small energy $\left(\Delta T / T_{c} \gtrsim 1\right)$ which create the circle(semicircle) with small effective radius $R \simeq \xi(T)$ and wide film $(w \gg \xi(T))$ the situation is different. In this limit the correction factor due to finite $w$ in Eqs. $(11,12)$ is small and one can see that $I_{d}$ is smaller for the photon absorbed on the edge than for the photon absorbed in the center of the film (due to difference in radiuses and in $\gamma$ which is finite). It correlates with our numerical results for low energy photons (small $\Delta T$ ) and wide film (see Fig. 6(a)).

We have to note that because of temperature gradient and proximity effect the distribution of the order parameter is nonuniform in the hot spot formed by the photon. This factor was not taken into account in above model and it brings the quantitative difference between our numerical and analytical results. Our numerical calculations show that at any considered photon energy the order parameter is finite in the hot spot at the moment when the first vortex-antivortex pair is nucleated. After nucleation the vortex and antivortex becomes immediately unbound and can move freely across the superconducting film. Therefore found above values for $I_{\text {pair }}$ and $I_{\text {res }}$ could be considered as low and upper thresholds for true detecting current.

\section{B. Regime with changing current}

In the experiments the circuity shown in Fig. 2 is usually used to prevent the latching of the superconductor in the normal state. As a result with appearance of the voltage drop over superconductor the current via the superconducting sample decreases and it switches back to the superconducting state.

In our calculations we use $R_{\text {shunt }}=50 \mathrm{Ohm}$. The kinetic inductance is evaluated using the following expression:

$$
L_{k}=\frac{4 \pi \lambda_{L}^{2} l}{w d}
$$

where $\lambda_{L}$ is a London penetration depth.

In Fig. $7(\mathrm{a})$ we present the time dependence of the voltage drop $V_{s}$ via the superconductor (resistance $R_{s}$ in Fig. 2) for two films with lengths $l=100 \mu m$ and 


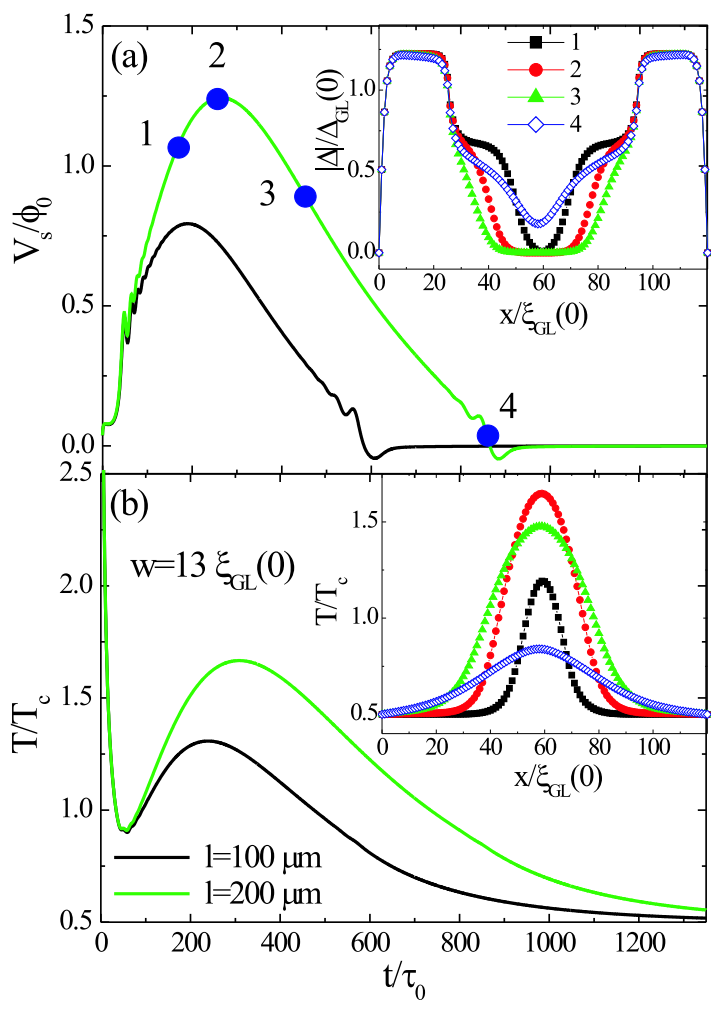

FIG. 7: (a) The time dependence of the voltage drop via the superconductive film calculated for two lengths: $l=100$ $\mu \mathrm{m}$ and $\mathrm{l}=200 \mu \mathrm{m}$. The inset shows the distribution of the magnitude of the order parameter along the film $($ at $y=0)$ at different moments in time. (b) The time dependence of the temperature in the center of the film (hot spot) for the same films. The inset shows the distribution of the temperature along the film $($ at $y=0)$ at different moments in time. The width of the film $w=13 \xi_{G L}(0)$, the bias current $I=0.6 I_{d e p}$, the local initial increase of the temperature $\Delta T=3.8 T_{c}(\lambda \simeq$ $3.9 \mu \mathrm{m})$.

$200 \mu \mathrm{m}\left(w=100 \mathrm{~nm}, d=4 \mathrm{~nm}\right.$ and $\left.\lambda_{L}=400 \mathrm{~nm}[19]\right)$. It is seen that with decrease of the kinetic inductance the duration and the amplitude of the voltage pulse becomes shorter and smaller correspondingly. The reason is simple - for smaller $L_{k}$ current via superconductor decreases faster, the temperature inside the normal domain increases slower (see Fig. 7(b)) and it takes less time to cool the sample up to bath temperature $T_{0}$ (see Fig. $7(\mathrm{~b}))$.

For the longest film the duration of the voltage pulse is about $900 \tau_{0}$ which is much larger than the typical time interval between consequent nucleation of the vortex-antivortex pair $\Delta t \simeq 10 \tau_{0}$ (we roughly estimate it as a time interval between nucleation of the second and third vortex-antivortex pairs - see Fig. 5). Therefore at least 90 vortex-antivortex pairs are nucleated during this voltage pulse.

In Fig. 8(a) we present the time dependence of $V_{s}$ for photons of different energy and position where it is absorbed. Notice that the shape of the voltage pulse and

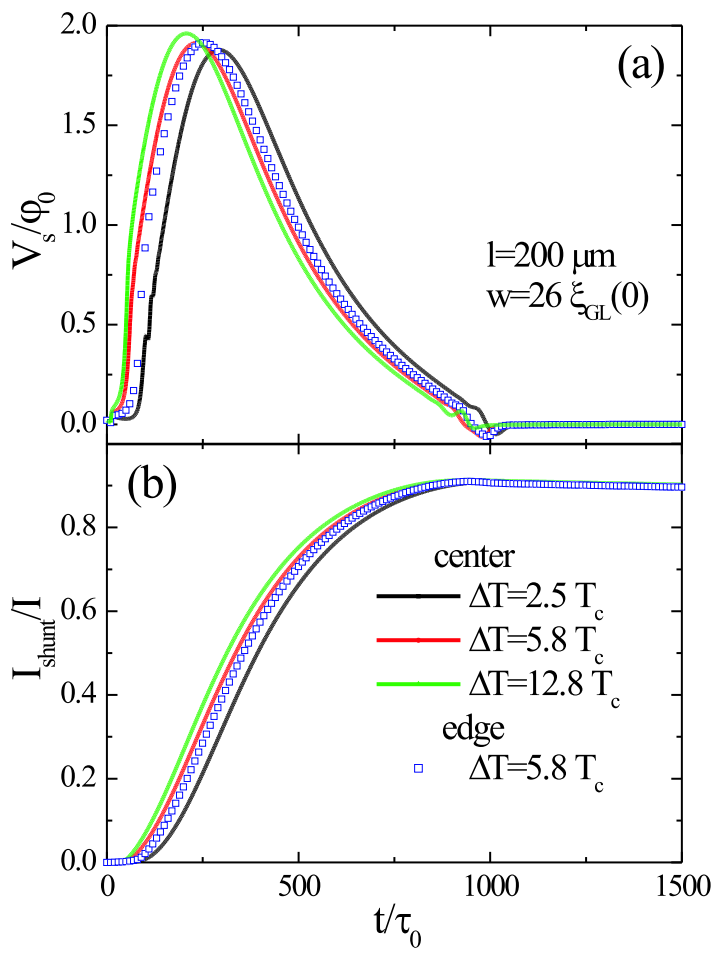

FIG. 8: (a) The time dependence of the voltage via the superconductive film with length $\mathrm{l}=200 \mu \mathrm{m}$ and width $w=$ $26 \xi_{G L}(0)$ for photons of different energy (different $\Delta T$ ) and position of the absorbtion (in the center or on the edge of the film). (b) The time dependence of the current via shunt resistance (the bias current $I=0.88 I_{\text {dep }}$ ).

current $I_{\text {shunt }}$ (see Fig. 8(b)) via shunt resistance (which is measured in the experiments with SSPD) slightly depend both on $\Delta T$ and absorption position. At times larger than $1000 \tau_{0}$ when the voltage drop via superconductor is equal to zero (see Fig. 8(a)) the current $I_{\text {shunt }}$ decays with characteristic time $\tau=L_{k} / R_{\text {shunt }} c^{2} \simeq$ $5 \cdot 10^{4} \tau_{0}$ for our choice of parameters.

We have to stress that our results for voltage pulse via superconductor (duration of the pulse, its amplitude) are obtained with neglecting the back action of the 'heated' phonons on the electrons. For our parameters $\left(\tau_{e-p h} \simeq 327 \tau_{0}\right)$ the duration of the pulse is about $3 \tau_{e-p h}$. But heating of the phonon subsystem occurs over time $t \gg \tau_{e-p h}$ [20]. Therefore we expect that hot phonons do not strongly influence the duration of the voltage pulse and its amplitude. And it certainly should not influence the value of the detecting current because it is determined from the stability of the superconducting state with respect to appearance of the single vortex or vortex-antivortex pair during evolution of the photon induced hot spot. 


\section{RELATION TO AN EXPERIMENT}

The model which we use (Eqs. (1-4)) is strongly oversimplified. First of all it does not take into account lose of the energy of photon at initial stage of formation of the hot spot on time scale $\tau_{e-e}$. Secondly we neglect temperature dependence of $C_{v}$, oversimplify the energy transfer to the phonons (last term in Eq. (2)) and we did not take into account possibility of direct partial destruction of the superconducting order parameter by incoming photon (in our approach $|\Delta|$ is influenced only via effective temperature of quasiparticles). Moreover, the time-dependent Ginzburg-Landau equation (Eq. (3)) is not quantitatively correct at temperatures lower than $\sim 0.9 T_{c}$ and (within quasiequilibrium approach) when $\tau_{e-e} \gtrsim \tau_{|\Delta|}$. Therefore direct quantitative comparison of our results with experiment (at least at low temperatures) looks speculative.

But despite this we believe that the used model catches the main physical mechanism of photon detection by current-carrying superconducting film which is the following. The incoming photon partially suppresses order parameter in the finite region. It leads to redistribution of the current density (supervelocity) in the film and such a state becomes unstable (without any fluctuations and if current is large enough but smaller than depairing current) with respect of appearance of the unbound vortexantivortex pair (if photon is absorbed far from edges) or single vortex (if photon is absorbed near the edge of the film). Lorentz force causes motion of these vortices that heats the film locally and gives rise to a voltage pulse.

We hope that our results could be used for understanding (not for direct fitting) of some experimental results. For example found dependence of the detection current on the position of the hot spot (see Fig. 6(a) and our analytical model) may be used for qualitative explanation of the monotonous decrease of the detection efficiency (DE) with the decrease of the energy of the incoming photon [7, 23, 24]. Indeed, if we fix the current (for example on the level $I=I_{d e p} / 2$ - see Fig. 6(a)) and start to decrease the energy of the photon, the edge region of the superconducting film first is "switched off" from the detection process (when $\Delta T \simeq 8 T_{c}$ which corresponds to $\lambda \simeq 1.9 \mu \mathrm{m}$ - see Fig. $6(\mathrm{a}))$ and at $\Delta T \simeq 5 T_{c}(\lambda \simeq 2.9 \mu \mathrm{m})$ the central region of the superconducting film stops to detect photons. As a result there is finite range of the wavelengths $\Delta \lambda \simeq 1 \mu m$ at $\left(I=I_{d e p} / 2\right)$ where the detection efficiency gradually changes (qualitatively such a behavior was observed in Refs. [7, 23, 24]).

If we fix the energy of photon then with increase of the current the central region of the film first starts to detect the photons and than the edge regions join the detection process. Therefore there is a finite interval of the currents $(\delta I)$ within which DE gradually grows with current increase (qualitatively such a behavior was observed in Ref. [25]). The real samples have different kinds of imperfections (variations of the thickness, width, material parameters, turns) having their own values of the detecting current and it obviously affects $\delta I$ at low currents. Our result show that even in ideal samples with no imperfections and at low temperatures (when effect of fluctuations is rather small) there will be finite $\delta I$ connected with presence of the edges. And our prediction is that the wider the sample the narrower is this interval of currents (see Fig. 6(a) and Eq. (12)).

\section{CONCLUSION}

In our work we use the quasiequilibrium approach and describe the deviation from the equilibrium in terms of the effective temperature of the quasiparticles which depends on time and coordinate. We assume that the absorbed photon creates initially the hot spot in the superconducting film with radius $R_{\text {init }}$ and local enhancement of the quasipartcile temperature by $\Delta T$ which is proportional to the energy of the photon. The temporal and spatial evolution of the effective temperature and superconducting order parameter in the superconductor we study by numerical solution of the time-dependent Ginzburg-Landau equation coupled with Poisson's equation for an electrical potential and heat diffusion equation.

We show that for photon of fixed energy the voltage response appears only at the current larger some critical one (we call it as detecting current $I_{d}$ ) when the vortexantivortex pair is nucleated in the center of the photon induced hot spot. Motion of the vortex and antivortex in opposite directions heats the superconductor and leads to the appearance of the growing normal domain when detecting current $I_{d}$ is about of depairing current. For high energy photon and narrow film $I_{d} \ll I_{d e p}$ the motion of the vortices-antivortices does not heat the superconductor and sample goes back to the superconducting state after nucleation of several vortex-antivortex pairs at $I \simeq I_{d}$ even in regime of constant current.

We find numerically that with increasing the width of the superconducting film $I_{d}$ increases and stays less than $I_{d e p}$ even for infinite film. We also find that the detecting current for photon absorbed on the edge of the film differs from $I_{d}$ for photon absorbed in the center of the film.

We develop simple analytical model to explain above results. We assume that absorbed photon creates in the superconducting film of finite width the region (in the form of circle or semicircle) where the superconducting order parameter $|\Delta|$ is partially suppressed and in framework of the London model we study the current redistribution and stability of the current-carrying state in such a system. We find that the superconducting vortex free state becomes unstable in the region with suppressed superconductivity at $I_{\text {pair }}<I_{d e p}$ and resistive state appears at larger current $I_{\text {pair }}<I_{\text {res }}<I_{d e p}$. Our analytical model predicts different values for the detecting current of the photon absorbed on the edge and in the center of the film in agreement with our numerical results. The last effect is connected with different redistribution 
of the supervelocity (current density) inside and outside the circle placed in the center of the film and the semicircle placed on the edge of the same film. We expect that our analytical results within the London model are valid for arbitrary temperatures contrary to the results based on the time-dependent Ginzburg-Landau and heat conductance equations which are strictly valid at $T>0.9 T_{c}$ and when $\tau_{e-e} \ll \tau_{|\Delta|}$ and $\tau_{e-e} \ll \tau_{e-p h}$.

To model operation of real superconducting single photon detector we consider the scheme with the resistance which is switched on in parallel to the detector and take into account the large kinetic inductance of real SSPD. We find that duration and amplitude of voltage pulse decrease with the decrease of the kinetic inductance while the detecting current practically does not change. We also find that the shape of the voltage pulse weakly depends on the energy of the absorbed photon and on the place of the absorbtion for the homogeneous film.

\section{Acknowledgments}

We acknowledge stimulating discussions with Alexei D. Semenov. This work was supported by the Russian Foundation for Basic Research and Russian Agency of Education under the Federal Target Programme "Scientific and educational personnel of innovative Russia in 2009-2013".
[1] G.N. Goltsman, O. Okunev, G. Chulkova, A. Lipatov, A. Semenov, K. Smirnov, B. Voronov, C. Williams, R. Sobolevski, Appl. Phys. Lett. 79, 705 (2001).

[2] A. Verevkin, J. Zhang, R. Sobolewski, A. Lipatov, O. Okunev, G. Chulkova, A. Korneev, K. Smirnov, G. Goltsman, A. Semenov, Appl. Phys. Lett. 80, 4687 (2002).

[3] M. Tarkhov, J. Claudon, J. Ph. Poizat, A. Korneev, A. Divochiy, O. Minaeva, V. Seleznev, N. Kaurova, B. Voronov, A. V. Semenov, G. Gol'tsman, Appl. Phys. Lett. 92, 241112 (2008).

[4] A. Divochiy, F. Marsili, D. Bitauld, A. Gaggero, R. Leoni, F. Mattioli, A. Korneev, V. Seleznev, N. Kaurova, O. Minaeva, G. Gol'tsman, K. G. Lagoudakis, M. Benkhaoul, F. Levy and A. Fiore, Nature Photonics, 2, 302 (2008).

[5] J. K. W. Yang, A. J. Kerman, E. A. Dauler, B. Cord, V. Anant, R. J. Molnar and K. K. Berggren, IEEE Trans. Applied Superconductivity 19, 318 (2009).

[6] H. Shibata, H. Takesue, T. Honjo and Y. Tokura, Appl. Phys. Lett. 97212504 (2010).

[7] M. Hofherr, D. Rall, K. Ilin, M. Siegel, A. Semenov, H. W. Hübers and N. A. Gippius, J. Appl. Phys. 108, 014507 (2010).

[8] A. D. Semenov, G. N. Gol'tsman, and A. A. Korneev, Physica C 351, 349 (2001).

[9] A. Semenov, A. Engel, H. W. Hübers, K. Il'in and M. Siegel, Eur. Phys. J. B 47, 495 (2005).

[10] A. Jukna, J. Kitaygorsky, D. Pan, A. Cross, A. Perlman, I. Komissarov, O. Okunev, K. Smirnov, A. Korneev, G. Chulkova, I. Milostnaya, B. Voronov, G. N. Gol'tsman, R. Sobolewski, Acta Physica Polonica A 113, 955 (2008).

[11] J. K. W. Yang, A. J. Kerman, E. A. Dauler, V. Anant, K. M. Rosfjord and K. K. Berggren, IEEE Trans. Applied Superconductivity 17, 581 (2007).

[12] A. Semenov, P. Haas, H. W. Hübers, K. Ilin, M. Siegel, A. Kirste, D. Drung, T. Schurig, and A. Engel, J. Mod.
Opt. 56, 345 (2009).

[13] A. J. Kerman, J. K. W. Yang, R. J. Molnar, E. A. Dauler, and K. K. Berggren, Phys. Rev. B 79, 100509(R) (2009).

[14] M. Tinkham, Introduction to superconductivity, (McGraw-Hill, NY, 1996).

[15] J. Zhang, W. Sysz, A. Pearlman, A. Verevkin, R. Sobolewski, O. Okunev, G. Chulkova, and G. N. Goltsman, Phys. Rev. B 67, 132508 (2003).

[16] A.M. Kadin, M. Leung and A.D. Smith, Phys. Rev. Lett. 65, 3193 (1990); A. M. Kadin and M. W. Johnson, Appl. Phys. Lett. 69, 3938 (1996).

[17] F. Giazotto, T. T. Heikkilä, A. Luukanen, A. M. Savin, and J. P. Pekola, Rev. Mod. Phys. 78, 217 (2006).

[18] L. Kramer and R.J. Watts-Tobin, Phys. Rev. Lett. 40, 1041 (1978).

[19] A. Kamplapure, M. Mondal, M. Chand, A. Mishra, J. Jesudasan, V. Bagwe, L. Benfatto, V. Tripathi and P. Raychaudhuri P, Appl. Phys. Lett. 96, 072509 (2010).

[20] A. D. Semenov, G. N. Gol'tsman and R. Sobolewski, Supercond. Sci. Technol. 15, R1 (2002).

[21] J. E. Mooij, in Percolation, Localization, and Superconductivity, edited by A. M. Goldman and S. A. Wolf (Plenum, New York, 1984), p. 325.

[22] H. Bartolf, A. Engel, A. Schilling, H.-W. Hübers and A. Semenov, Phys. Rev. B 81, 024502 (2010).

[23] A. D. Semenov, P. Haas, H. W. Hübers, K. Ilin, M. Siegel, A. Kirste, T. Schurig, and A. Engel, Physica C 468, 627 (2008).

[24] L. Maingault, M. Tarkhov, I. Florya, A. Semenov, R. Espiau de Lamaëstre, P. Cavalier, G. Gol'tsman, J. P. Poizat, and J. C. Villégier, J. Appl. Phys. 107, 116103 (2010).

[25] B. Baek, A. E. Lita, V. Verma and S. W. Nam, Appl. Phys. Lett. 98, 251105 (2011). 